\title{
Computational Analysis of Hip Implant Surfaces
}

\author{
A. Vulović ${ }^{1,2,3^{*}}$, N. Filipović ${ }^{1,2}$ \\ ${ }^{1}$ Faculty of Engineering, University of Kragujevac, 6 Sestre Janjić Street, 34000 Kragujevac, \\ Serbia \\ e-mail: aleksandra.vulovic@kg.ac.rs, fica@kg.ac.rs \\ ${ }^{2}$ Bioengineering Research and Development Center (BioIRC), 6 Prvoslava Stojanovića Street, \\ 34000 Kragujevac, Serbia \\ ${ }^{3}$ Steinbeis Advanced Risk Technologies Institute doo Kragujevac, 25 Kneza Milosa Street, \\ Kragujevac, Serbia \\ *corresponding author
}

\begin{abstract}
During the total or partial hip replacement procedure, a damaged part of the hip joint is replaced with an artificial one. Both types of procedures include removal and replacement of the femoral head. After the femoral head is removed, an implant is inserted into the hollow femur. When the healing process starts, the newly formed bone interlocks with the inserted implant. Experimental studies indicate that lower shear stress in the bone-implant contact will result in better boneimplant connection. The goal of this study was to use the numerical approach in order to analyze the shear stress values of the three simple modifications of the hip implant surface. Numerical approach consisted of the implementation of the finite element method (FEM) for the simulation of the bone-implant interaction and for the calculation of the shear stress values of the three modified surface topographies in the titanium alloys (Ti-6Al-4V) implants.
\end{abstract}

Keywords: finite element method, hip implant, shear stress, surface modification

\section{Introduction}

When a hip joint is damaged or worn out, total or partial hip replacement procedure is performed. During this procedure, a damaged part of the hip joint is replaced with appropriate artificial one (Derar and Shahinpoor, 2015). Both types of hip replacement procedures include removal and replacement of the patient's femoral head. After the femoral head is removed, a metal stem is inserted into the hollow femur. This is considered to be one of the most successful procedures in the world. Annually, more than one million hip replacement surgeries are performed worldwide, while it is anticipated that this number will double in the next decade (Shan et al. 2014). It is considered to be a routine procedure with a minimum rate of early complications.

Although early complications are rare, the biggest issue with this procedure is the limited life span of the implant. Average implant life span is about 15 years, while about $10 \%$ of patients need to have revision surgery within 10 years (Sargeant and Goswami, 2006). Aseptic loosening is one of the major causes of revision surgeries. A possible way to reduce the number of revision 
surgeries as a result of aseptic loosening is to provide a better bone-implant fixation, which means reducing the micro movements between the bone and the implant.

When an implant is inserted into the fractured bone, the osseointegration starts to happen. The osseointegration represents the direct functional connection between the inserted implant and bone. In order to improve the osseointegration, an implant with a rough surface should be used (Mattila et al. 2009; Halldin et al. 2015). This connection between an implant and a bone is commonly analyzed using in vivo experiments, which indicate that the increased interfacial shear strength can be obtained by the increased surface roughness of cylindrical implants (Loberg et al. 2010). During the previous years, a number of experimental studies have been done with goal to analyze the effect of surface texturing of orthopaedic implants. Several papers have analyzed the effect of the concave dimples. Ito et al. (2000) have investigated if forming of the concave dimples on the surface of the metal femoral head could reduce the possibility of polyethylene wear. Zhou et al. (2012) have analyzed the influence of concave dimples on the metallic counterface on the wear of ultra-high molecular weight polyethylene. According to the mathematical model by Hannson and Norton (1991), rough surface leads to the increase in the interfacial shear strength. Using the numerical approach, Mattila et al. (2009) showed a shear stress distribution for a nonresorbable fibre reinforced composites during static load while bone and implant were rigidly bonded. An improved approach is presented in paper by Halldin et al. (2015), where better boundary conditions and geometry were used.

The goal of the present study was to obtain shear stress values for three different modified hip implant surfaces. The modification of the surface was done with spherical geometries. The shear stress values were obtained using the finite element method. This method has been used for the design and the analysis of orthopaedic devices, as this approach complements experimental work in a way that can provide information that cannot be obtained otherwise.

The rest of the paper is organized as follows. Created models, their material properties and applied boundary conditions are described in section 2 . Section 3 covers the results of the finite element analysis and the discussion, while the conclusion is given in section 4.

\section{Materials and Methods}

In order to carry out numerical simulations of different implant surface modification several steps need to be followed: creating the 3D models and their discretization, defining appropriate material properties, defining appropriate boundary conditions, analysing the problem using the finite element method.

\subsection{Geometries}

Three models have been created. We have considered the interaction between the cementless femoral implant and the femoral bone. The focus was on their interaction in the femoral shaft, which is the reason why all created models have only two different layers, one corresponding to femoral implant and the other corresponding to cortical bone.

The analyzed models were created using a computer aided design (CAD) software. Each layer of every model was exported from CAD software as .stp file which was then used for mesh creation. Model meshing was performed manually.

Medium height of rough surface was around $2 \mu \mathrm{m}$ depending on the model. Considering that this value is thousand times smaller compared to dimensions of implant and bone we have decided to focus only on the small area outside the interaction zone. Created models are presented in the following figures (Fig. 1-3). 


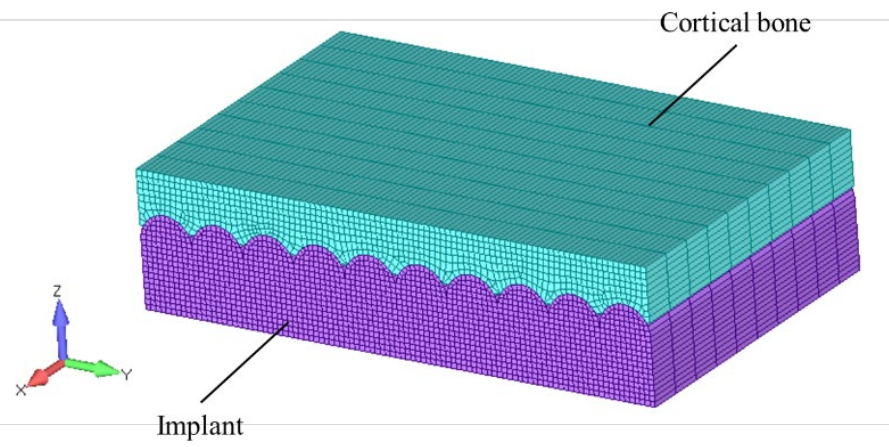

Fig. 1. Model 1.

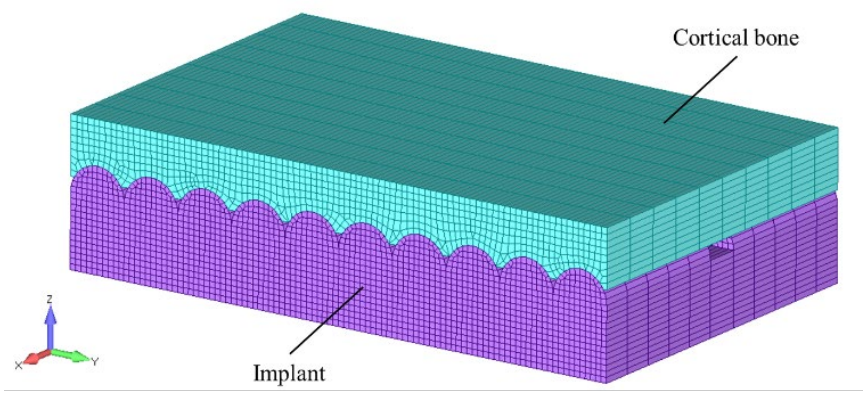

Fig. 2. Model 2.

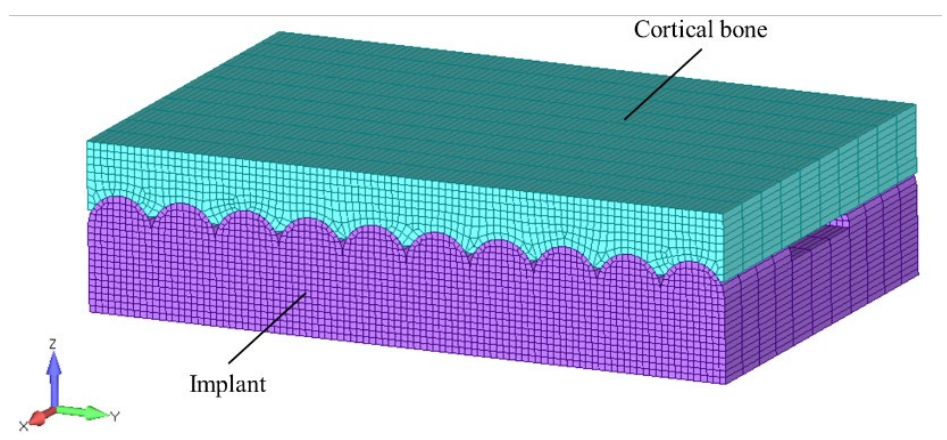

Fig. 3. Model 3.

The total number of nodes and elements per model is given in Table 1.

\begin{tabular}{|c|c|c|}
\hline Model & \multicolumn{2}{|c|}{ Number of Nodes and Elements } \\
\cline { 2 - 3 } Number & Nodes & Elements \\
\hline Model 1 & 30008 & 24780 \\
\hline Model 2 & 34264 & 26080 \\
\hline Model 3 & 33284 & 25160 \\
\hline
\end{tabular}

Table 1. Number of nodes and elements per model 
Length, width and height of the implant and bone model were the same for all models. The only difference between the models was in the distance between two half cylinders. For the first model, there was only one half-cylinder along the width of the model, while for the models 2 and 3 there were two half cylinders with different distance between them. Dimensions of the created models are presented in Table 2.

\begin{tabular}{|c|c|}
\hline Dimension & Value \\
\hline Implant model length & $80 \mu \mathrm{m}$ \\
\hline Implant model width & $50 \mu \mathrm{m}$ \\
\hline Implant model height & $10 \mu \mathrm{m}$ \\
\hline Bone model length & $80 \mu \mathrm{m}$ \\
\hline Bone model width & $50 \mu \mathrm{m}$ \\
\hline Bone model height & $14 \mu \mathrm{m}$ \\
\hline Half-cylinder radius & $4 \mu \mathrm{m}$ \\
\hline
\end{tabular}

Table 2. Dimensions used for the models

All created models were scaled 1000 times.

\subsection{Material properties}

As previously explained, two materials were used for the finite element analysis. The used material properties are defined to describe behaviour of cortical bone and hip implant material. In order to simplify simulations, material properties of cortical bone were considered to be isotropic, homogeneous and linear elastic instead of orthotropic or anisotropic. For the analyzed hip implant titanium alloy Ti6A14V was chosen. Applied material properties of cortical bone and hip implant were taken from literature and the values are shown in Table 3.

\begin{tabular}{|c|c|c|c|}
\hline Material & $\begin{array}{c}\text { Young's modulus } \\
{[\text { [GPa] }}\end{array}$ & $\begin{array}{c}\text { Poisson } \\
\text { ratio }\end{array}$ & Reference \\
\hline $\begin{array}{c}\text { Cortical } \\
\text { bone }\end{array}$ & 16.7 & 0.3 & $\begin{array}{c}\text { Aradhya and Doddamani, } \\
2015\end{array}$ \\
\hline Implant & 109 & 0.34 & Das and Sarangi, 2014 \\
\hline
\end{tabular}

Table 3. Applied material properties

The work of Peng et al. (2006) indicated that the use of isotropic instead of orthotropic material properties for femoral bone does not show much difference of the results under singleleg standing which corresponded to the used boundary conditions.

\subsection{Boundary conditions}

The load we have used for the analysis of the hip implant surfaces, was obtained from the simulation of interaction between femoral bone and hip implant without modifications under walking static condition (Chalernpon et al. 2015). The obtained results from this simulation were used as the input for the analysis of the modified implant surfaces.

The load and constraints used for the simulation of implant without modification were adapted from Chalernpon et al. (2015). Applied boundary conditions are presented in Fig. 4. 


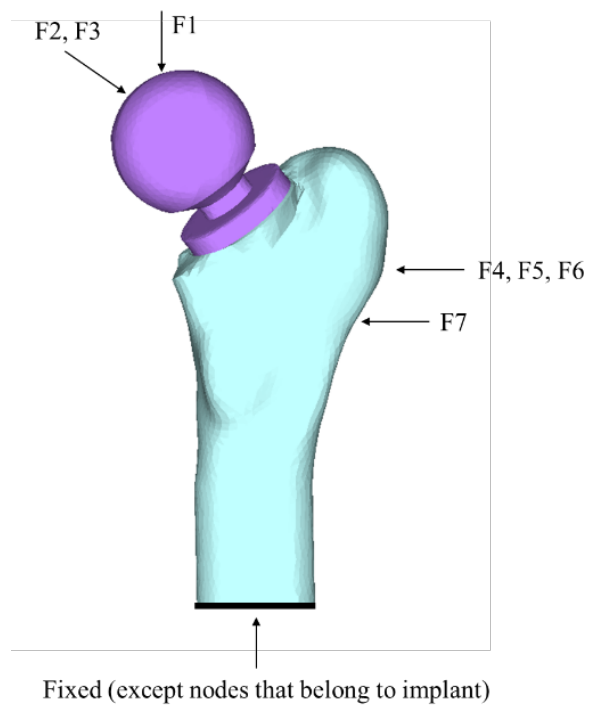

Fig. 4. Applied boundary conditions for model without implant modifications.

Used forces included body weight as well as the appropriate muscle forces. Explanation for applied forces is given in Table 4.

\begin{tabular}{|c|c|}
\hline Position & Force \\
\hline F1 & Body weight \\
\hline F2 & Hip contact \\
\hline F3 & Intersegmental resultant \\
\hline F4 & Abductor \\
\hline F5 & $\begin{array}{c}\text { Tensor fascia latae, proximal } \\
\text { part }\end{array}$ \\
\hline F6 & part \\
\hline F7 & Vastus lateralis \\
\hline
\end{tabular}

Table 4. Applied forces

The implant was in contact with the cortical bone. In order to simulate micro motions that can occur between cortical bone and implant, contact with friction between elements was defined. For this study, we have used the friction coefficient of 0.39 obtained from the experimental study in which the tribological behaviour of Ti6Al4V against cow bone was investigated (Das and Sarangi, 2014).

The constraints applied to the models with modified implant surfaces were adapted from Halldin et al. (2015). The upper surface of the cortical bone was fixed, while the sideways of the implant were allowed to move in the $\mathrm{y}$ and $\mathrm{z}$ direction (locked in the $\mathrm{x}$ direction) and the bottom surface of the implant was locked in the $\mathrm{z}$ direction. All the other elements were allowed to move in all three directions. 
For the modified implant models, same contact condition was used as in the model without modification.

\section{Results and discussion}

As explained in Section 2, the results obtained from the simulation of the interaction between femoral bone and implant without modification were used as the load for the modified models. We have chosen to use the total contact force as the input for the simulation of models with modifications.

The shear stress distribution for the first model can be seen in Fig. 5. The calculated shear stress values were in the range from 0.0169 to $4.145 \mathrm{MPa}$.
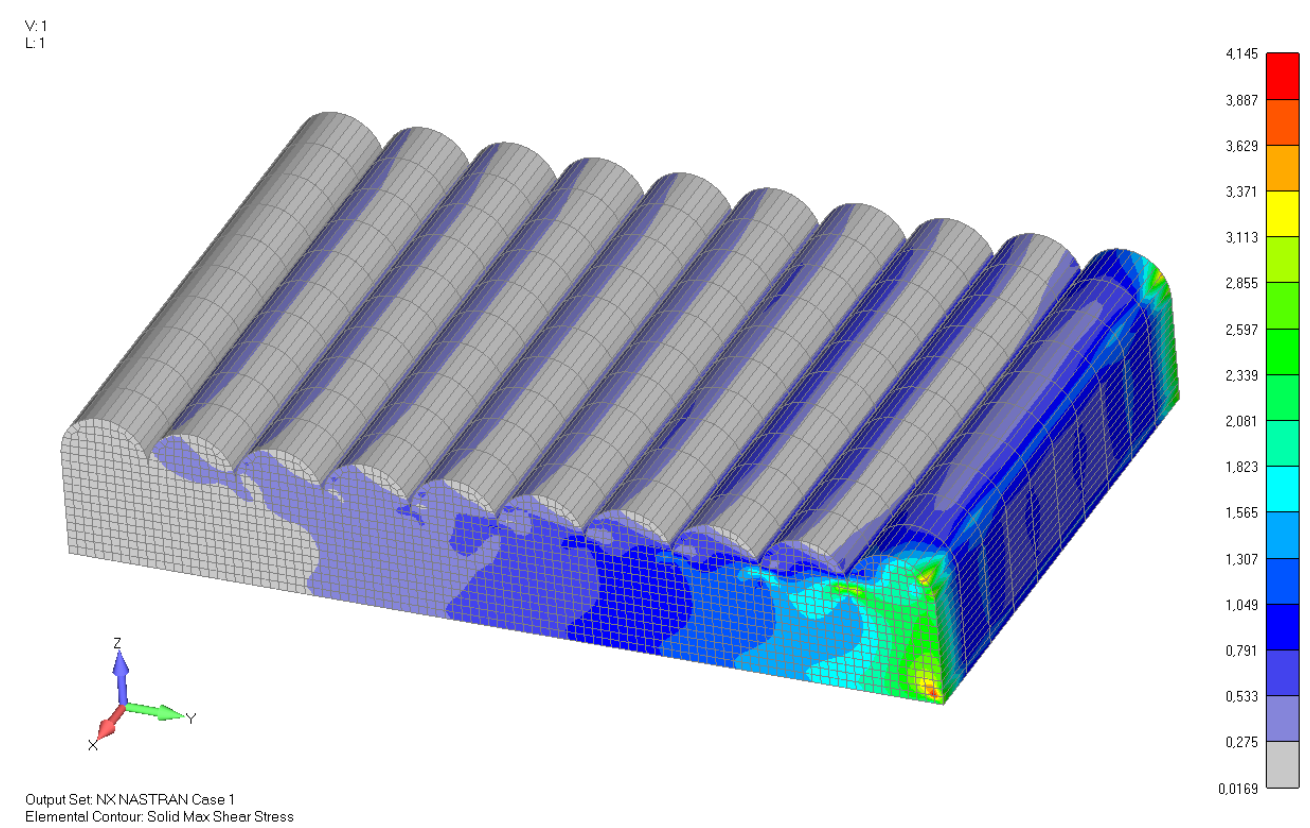

Fig. 5. Shear stress distribution (in MPa) for model 1.

It can be noticed that the maximum value was located on the implant's side and not on the modified surface. The highest shear stress values were calculated closest to the right side of the model, where the contact force was defined. The shear stress values decreased going from the right to the left side of the model. On the left side of the model, the calculated shear stress values were almost $0 \mathrm{MPa}$. Considering only the surface modifications, the majority of the surface had the shear stress that was lower than $1 \mathrm{MPa}$.

The shear stress distribution for the model 2 can be seen in Fig. 6. The calculated shear stress values for the second model were in the range from 0.0169 to $5.023 \mathrm{MPa}$. 


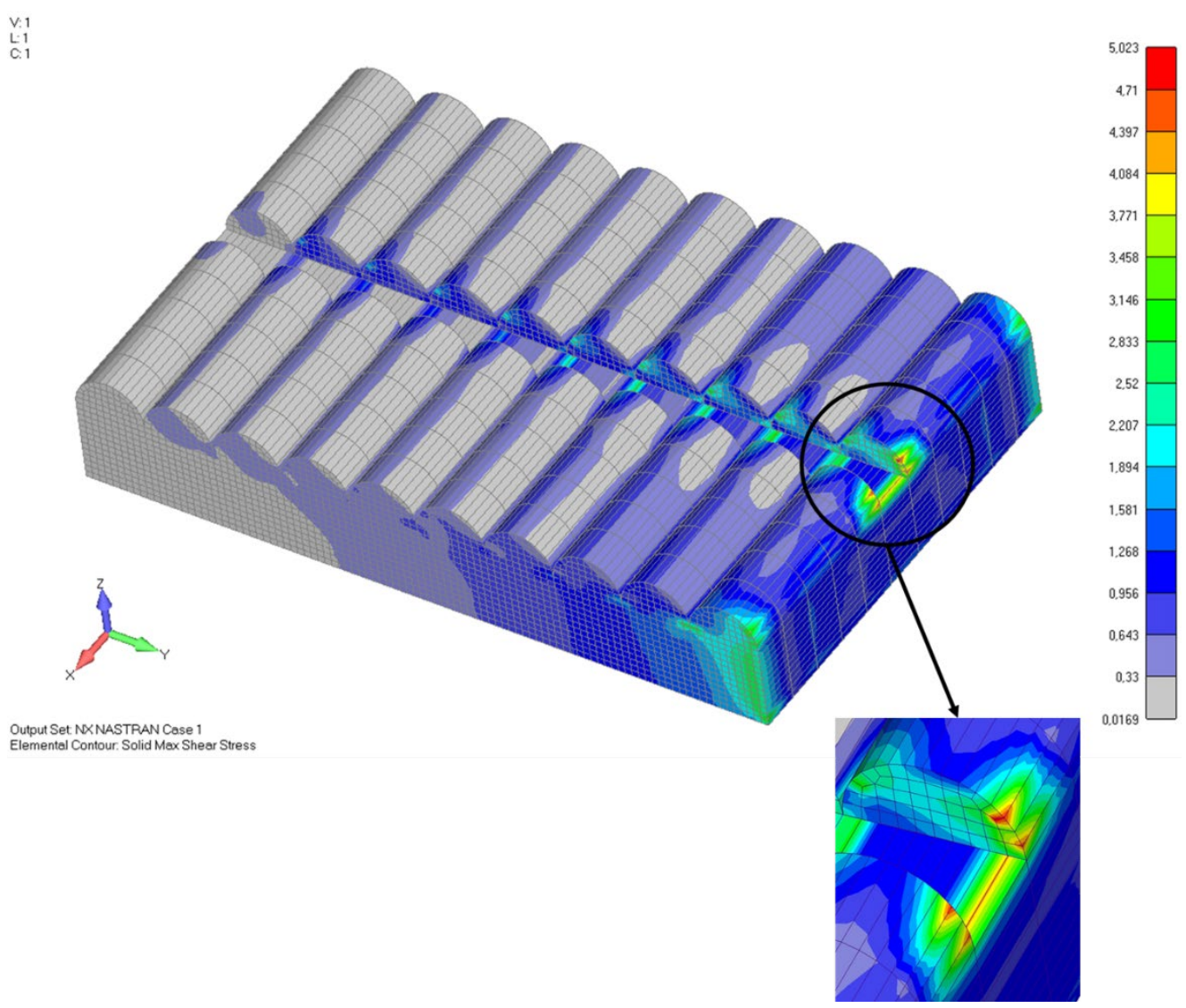

Fig. 6. Shear stress distribution (in MPa) for model 2.

It can be noticed that not having one half cylinder per model's width had the effect on the shear stress distribution. In this case, the maximum value was not located on the implant side. As it can be seen in Fig. 6, the highest shear stress was calculated to be on the surface modification that was closest to the right side of the model, where the contact force was defined. As in the previous model, the shear stress values decreased going from the right to the left side of the model.

In the case of the second model, the shear stress values on the modified implant surface were higher compared to the first model, where the majority of the surface had the shear stress lower than $1 \mathrm{MPa}$. It can be noticed that not having one half cylinder per width leads to higher shear stress values in the places of connections between half cylinders. Compared to the first model where only the four half cylinders had shear stress above $1 \mathrm{MPa}$, here only the last half cylinder had shear stress lower than $1 \mathrm{MPa}$.

The shear stress distribution for the model 3 can be seen in Fig. 7. The calculated shear stress values were in the range from 0.017 to $9.095 \mathrm{MPa}$. 

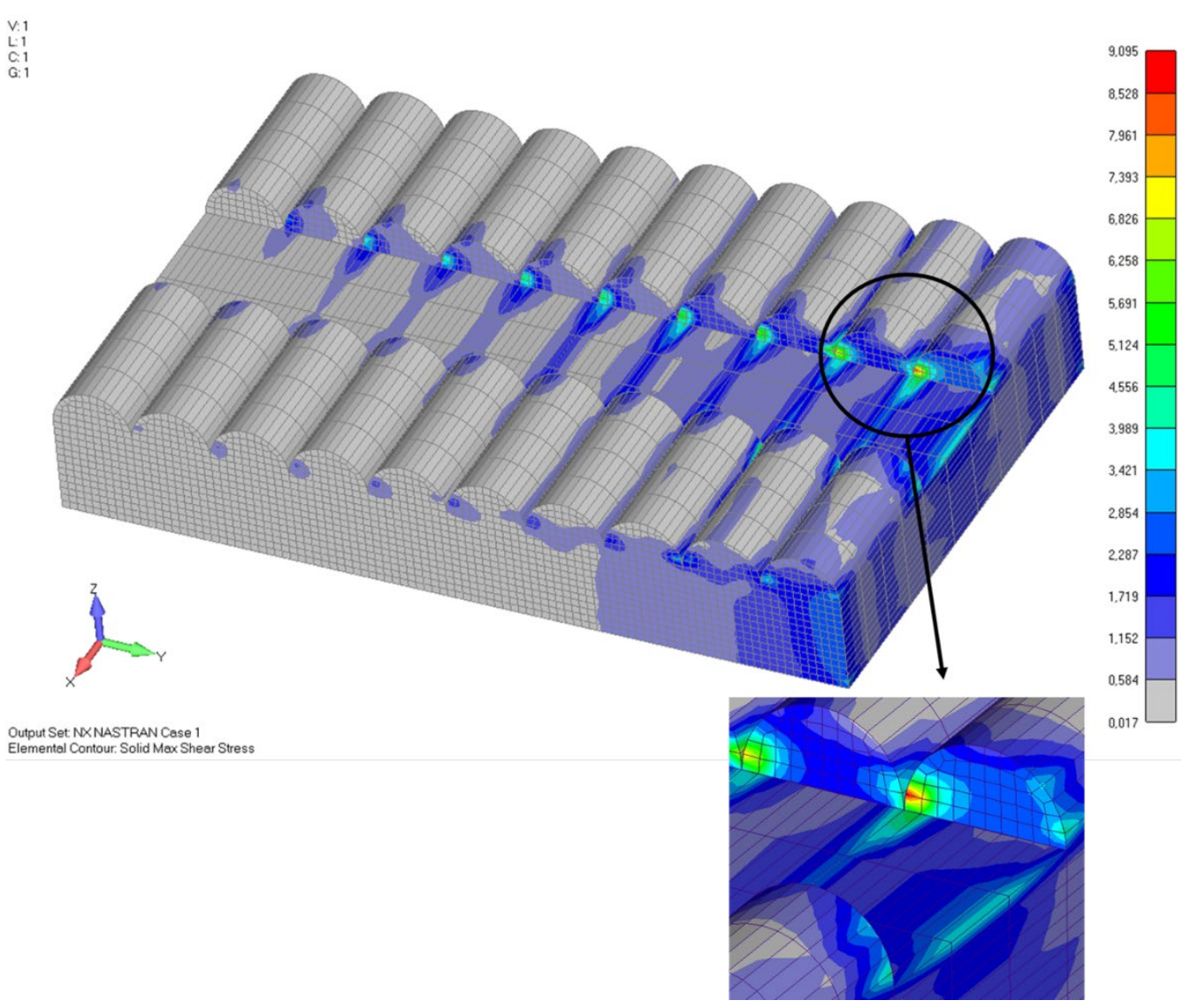

Fig. 7. Shear stress distribution (in $\mathrm{MPa}$ ) for model 3.

The distribution of the shear stress for the third model was closer to the second model. As in the previous model (model 2) the maximum value was not located on the implant side. Again, the highest shear stress was calculated to be on the surface modification that was closest to the right side of the model, where the contact force was defined. However, in this case, the highest value was on the side closer to second half cylinder. It can be noticed that only inner ends of the half cylinders have significant shear stress values.

Comparison of the shear stress distribution for all three models can be seen in Fig. 8. The models were cut in the middle with YZ plane. 


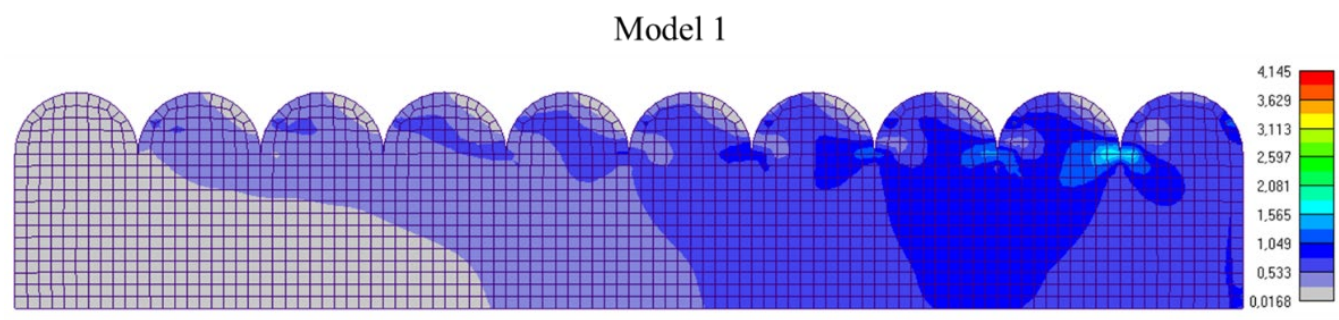

Model 2

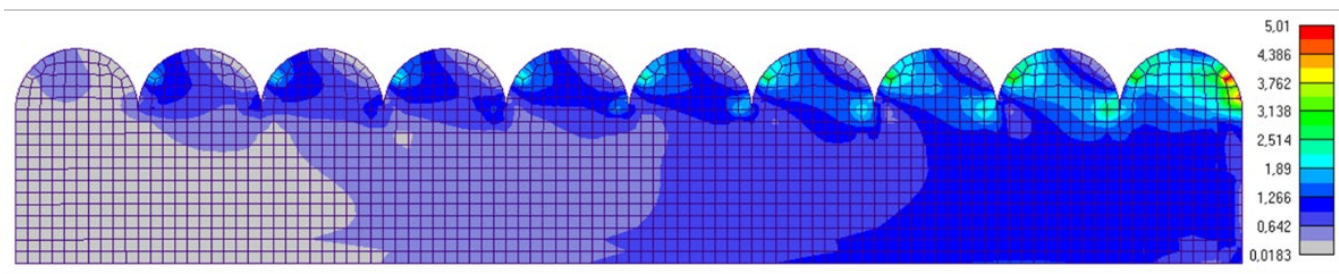

Model 3

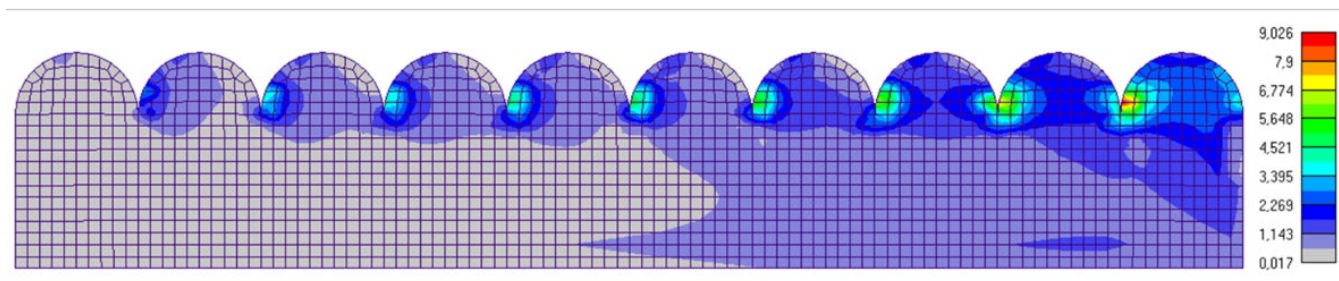

Fig. 8. Comparison of shear stress distribution for cross section (YZ plane).

Considering the presented results, it can be noticed that the length of the half-cylinder has the effect on the shear stress values and distribution. Smaller length led to higher shear stress results which can be noticed by comparing the results from previous three models. The maximum shear stress values for each model are given in Table 5 .

\begin{tabular}{|c|c|}
\hline Model & $\begin{array}{c}\text { Maximum shear } \\
\text { stress value [MPa] }\end{array}$ \\
\hline Model 1 & 4.145 \\
\hline Model 2 & 5.023 \\
\hline Model 3 & 9.095 \\
\hline
\end{tabular}

Table 5. Comparison of the maximum shear stress values

According to Mattila et al. (2009), shear stress at the implant-bone interface should be minimized in order to promote bone ingrowth. Based on this criterion, considering both the maximum shear stress value and the distribution of the shear stress in the present models we can conclude that the model 1 would be the best option for the modification of the implant surface. 


\section{Conclusions}

The presented results are a promising step forward in our research of the modified hip implant surfaces. However, we are aware that the shear stress results obtained using the numerical approach are not realistic in terms that they do not apply to every single person. The forces used for this simulation (both for the load of the complete implant-bone model and the load used for the modified implants) change from person to person depending on person's weight. Also, each human body is different, and each will have different reaction to inserted implant, which is something we cannot know based on the simulations.

However, this approach provides us with results that we would not be able to obtain otherwise. In the industry of orthopaedic implants, there is a growing need for numerical simulations in order to obtain stress values, such as shear stress and maximum principal stress. Based on the information we are able to obtain using finite element analysis, we could potentially create guidelines for the best topography for each type of implant (i.e. hip, dental, etc.) according to the ideal conditions.

Acknowledgements This paper has received funding from the European Union's Horizon 2020 research and innovation programme under grant agreement No. 760921 - PANBioRA. This article reflects only the author's view. The Commission is not responsible for any use that may be made of the information it contains. The research has also been carried out with the support of the Ministry of Education, Science and Technological Development of the Republic of Serbia by projects III41007 and OI174028.

\section{References}

Aradhya K S S, Doddamani M R (2015). Characterization of Mechanical Properties of SiC/Ti6Al-4V Metal Matrix Composite (MMC) Using Finite Element Method, American Journal of Materials Science, 5(3C), 7-11.

Chalernpon K, Aroonjarattham P, Aroonjarattham K (2015). Static and dynamic load on hip contact of hip prosthesis and Thai femoral bones, International Journal of Medical, Health, Biomedical, Bioengineering and Pharmaceutical Engineering, 9, 251-255.

Das S, Sarangi S K (2014). Finite Element Analysis of Femur Fracture Fixation Plates, International Journal of Basic and Applied Biology, 1(1), 1-5.

Derar H, Shahinpoor M (2015). Recent Patents and Designs on Hip Replacement Prostheses, The Open Biomedical Engineering Journal, 9, 92-102.

Halldin A, Ander M, Jacobsson M, Hansson S (2015). Simulation of the mechanical interlocking capacity of a rough bone implant surface during healing, BioMed Eng OnLine, 14, 45.

Hansson S, Norton M (1999). The relation between surface roughness and interfacial shear strength for bone-anchored implants. A mathematical model, Journal of Biomechanics, 32, 829- 836.

Ito H, Kaneda K, Yuhta T, Nishimura I, Yasuda K, Matsuno T (2000). Reduction of polyethylene wear by concave dimples on the frictional surface in artificial hip joints, The Journal of Arthroplasty, 15, 332-338.

Loberg J M I, Hansson S, Ahlberg E (2010). Characterisation of titanium dental implants I: Critical assessment of surface roughness parameters, The Open Biomaterials Journal, 2, 18.

Mattila R H, Laurila P, Rekola J, Gunn J, Lassila L V, Mäntylä T, Aho A J, Vallittu P K (2009). Bone attachment to glass-fibre- reinforced composite implant with porous surface, Acta biomaterialia, 5, 1639-1646. 
Peng L, Bai J, Zeng X, Zhou Y (2006). Comparison of isotropic and orthotropic material property assignments on femoral finite element models under two loading conditions, Medical Engineering \& Physics, 28, 227-233.

Sargeant A, Goswami T (2006). Hip implants: Paper V. Physiological effects, Materials and Design, 27(4), 297-307.

Shan L, Shan B, Graham D,Saxena A (2014). Total hip replacement: a systematic review and meta-analysis on mid-term quality of life, Osteoarthritis and Cartilage, 22(3), 389-406.

Zhou X, Galvin A L, Jin Z, Yan X, Fisher J, (2012) The influence of concave dimples on the metallic counterface on the wear of ultra- high molecular weight polyethylene, Proc. Inst. Mech. Eng., Part J: J. Eng. Tribol,. 226, 455-462. 